\title{
Abriendo camino en los entornos digitales de comunicación: algunas propuestas significativas
}

\author{
Juan Carlos MARCos ReciO \\ jmarcos@ucm.es \\ Manuel FERNÁNDEZ SANDE \\ manuel.fernandez@ccinf.ucm.es \\ Facultad de Ciencias de la Información \\ Universidad Complutense de Madrid
}

\begin{abstract}
"Robot reporters also make sense on the business end: By using software to create content, publishers get lots of cheap monetizable content without having to employ any more expensive humans".
\end{abstract}

Ricardo Bilton (Verificación de la realidad: los robots no van a escribir las noticias).

Recibido: 15/02/2014

Aceptado: 20/03/2014

\section{RESUMEN}

Los formatos digitales en los medios de comunicación, especialmente en los periódicos, atraviesan por una fase de nuevos modelos comunicativos. Se hace un recorrido por el mundo digital, sus cifras más significativas y algunos estudios que avalan el crecimiento en los entornos digitales, especialmente las referidas a los medios de comunicación. Además, se proponen algunos proyectos de aplicación inmediata en formato digital y con una temática específica que no suelen cubrir los medios tradicionales. Al frente de estos proyectos figuran expertos periodistas, con más de dos décadas en puestos de responsabilidad y analistas e inversores en contenidos digitales.

Palabras clave: Periódicos, Entornos digitales, Comunicación, Proyectos mediáticos, Medios digitales, Audiencias.

\section{Leading the way in digital communication environments: some significant proposals}

\begin{abstract}
Digital formats in the media, especially newspapers, are going through a phase of new communication models. A tour of the digital world, its most significant figures and some studies supporting the growth in digital environments, especially those concerning the media is made. In addition, some projects for immediate implementation in digital format with a specific theme, which usually do not cover traditional media are proposed. At the forefront of these projects include expert journalists, with more than two decades in positions of responsibility and analysts and investors in digital content.
\end{abstract}

Keywords: Newspapers, Digital Environments, Communication, Media Projects, Digital Media, Audiences. 


\section{INTRODUCCIÓN}

En los diez primeros años del siglo actual han sucedido más y mayores transformaciones en los medios de comunicación que a lo largo de los últimos tres siglos. No fueron cambios radicales porque el siglo XX fue quien allanó ese camino. Mejoras en los sistemas de redacción, impresión, maquetación y diseño y, en general, en la distribución hicieron que el producto periódico impreso se convirtiera en el principal medio de comunicación en competencia con la radio y la televisión. La noticia impresa seguía siendo el referente informativo, apoyado en los soportes documentales y las investigaciones periodísticas.

El siglo XX fue considerado el de la información. Cualquier toma de decisiones requería una búsqueda. El mundo financiero, la educación, el arte y la cultura, los propios medios de comunicación eran portadores de información. Se consideraba a la sociedad suficientemente preparada para el manejo de los contenidos que circulaban a través de los medios. Y antes de que se acabara se produjo la gran transformación en la sociedad, ya que se juntaron dos palabras para cambiar el orden hasta entonces establecido y una de ellas era precisamente esa: tecnologías de la información.

No salieron de la nada. Ya en los años ochenta del siglo pasado, muchos investigadores accedían a la información a través de bases de datos. Los contenidos impresos tenían un "hermano mayor" dentro de las incipientes redes que entonces se iban constituyendo. También en los medios de comunicación se empieza a trabajar con documentos en línea como el Information Bank del The New York Times en los años 80.

Entonces llegó Internet y con ella el paso hacia un nuevo orden en la sociedad. También en los medios de comunicación. Un lugar donde la tecnología se comparte y donde lo difícil para el usuario se convierte en material manejable para crear, seleccionar y difundir mensajes propios o de terceros, con el fin de ser protagonista de su vida y de la de otros.

La gran mayoría de los medios de comunicación tardaron en darse cuenta de que la información tenía otro valor. Aferrados a un negocio centenario, muchos periódicos impresos apostaron tímidamente por un producto digital, de parecidas características a su versión impresa. Primer fracaso absoluto. La presión de los lectores haría luego el resto. Acuciados por nuevos medios, creados ya en entornos digitales, los grandes medios de comunicación que habían sido referentes en la información, ofrecieron sus contenidos en nuevas páginas web y plataformas gestionadas con publicidad para reportar algunos ingresos. Desde entonces, se probaron varios métodos: redacciones separadas, redacciones integradas, nuevos formas de publicar y sobre todo el periodismo ciudadano, el periodismo participativo, creándose medios propios o apoyados en grandes cabeceras, como el caso de The Huffington Post, un periódico en línea y blog agregador de noticias, fundado por Arianna Huffington, Kenneth Lerer y Jonah Peretti. 
En los últimos diez años se ha escrito mucho sobre este tipo de periodismo, sobre medios digitales, etc., pero no tanto como en los años 60 con el llamado nuevo periodismo ${ }^{1}$. Se trata de un tipo de informaciones publicadas en Estados Unidos en una época de importantes cambios sociales y culturales. Años de conflictos, de guerras, de movimientos sociales que toman como referencia libros como: "A sangre fría" de Truman Capote, artículos y textos de Tom Wolfe, y trabajos periodísticos y reportajes con la firma del escritor Gay Talese.

En la era digital una búsqueda sencilla en Google arroja aproximadamente 1.190.000 resultados sobre el concepto Nuevo Periodismo, frente a los aproximadamente 736.000 resultados cuando se pide información sobre Periodismo Digital. Así pues, aún queda margen para publicar y eso que en la actualidad el número de escritores, de periodistas, de personas comprometidas con la escritura en los medios ha ido creciendo exponencialmente.

\section{METODOLOGÍA}

Con frecuencia en estos tiempos digitales se vuelve la vista atrás y se muestran estudios comparativos de lo que fueron, son y serán los medios de comunicación impresos en su devenir hacia lo digital. Pero, en esta ocasión, el artículo obedece a un estudio y seguimiento bibliográfico durante el año 2013, especialmente en los últimos meses, sobre proyectos que se están poniendo en marcha para entornos digitales y la evolución de la última década. Se trata de buscar un nicho de mercado, aquél en el que los grandes medios de comunicación apenas inciden, y ofrecer informaciones precisas, actualizadas y de interés para lectores de comunidades grandes en número porque se publican en Internet, pero con una especialidad. Así, la mayoría de estos proyectos abarcan un área temática, por ejemplo, legislación, derecho, tribunales, etc. El formato periodístico ofrece informaciones actualizadas, con numerosas declaraciones y apoyos documentales.

No se trata sólo de analizar y estudiar uno o varios medios digitales. Son apuestas serías, rigurosas, controladas en lo comercial y financiero, y con un equipo reducido de redactores especializados en un área. En los ejemplos que se proponen, la mayoría de los medios están encabezados por periodistas con un largo recorrido en medios de primer nivel, con más de una veintena de años ejerciendo la profesión y con cargo en periódicos de la talla del The New York Times, The Washington Post, etc. Convencidos de que existen nuevas fórmulas para publicar y lectores agradecidos que esperan ese tipo de información, las alternativas a los grandes medios de información están servidas. Queda comprobar si se perpetuarán o serán más bien una moda pasajera.

${ }^{1}$ Especialmente significativo para los periodistas fue el libro de Tom Wolfe: "El nuevo periodismo". Madrid: ANAGRAMA, 2000. ISBN 9788433912022 
Es objeto de este artículo mostrar un panorama general sobre la situación de los medios de comunicación, tomando como referencia la primera década del siglo actual. Además, y esta sería la parte novedosa, se ofrecen algunos avances sobre medios que están experimentando nuevos marcos informativos, abriendo caminos a la comunicación con ejemplos de empresas y personas que con una gran experiencia en los medios, apuestan ahora por otros formatos periodísticos. Son medios que han descubierto una línea informativa "desatendida" o poco cuidada por los grandes medios generalistas.

\section{EL MUNDO DIGITAL EN CIFRAS: ALGUNOS DATOS EN MEDIOS}

Antes de que existiera el periodismo, que no la comunicación, las bibliotecas eran el referente educativo, cultural e informativo. Es una obligación de bibliotecas y centros de documentación la búsqueda, selección, análisis y conservación de libros y documentos con el fin de que los usuarios puedan hacer uso de los mismos y la ciencia avance al disponer de suficiente información. Pasaron varios siglos, más de la mitad de la historia conocida del ser humano, sin que hubiera problemas para acceder al contenido, por entonces escaso. Los productores (autores) eran pocos y en la mayoría de las veces alejados unos de otros; y los lectores (usuarios) mostraban poco interés en lo que se publicaba.

Nunca hasta finales del siglo XX se tuvo la necesidad de cuantificar la información. Es cierto que en los siglos pasados, las bibliotecas nacionales crearon sus bibliografías para disponer de las principales publicaciones con las que contaban. Eran trabajos cuantificables, sin duda, pero pretendían hacer listados de esas obras, no tanto las consultas por parte de los investigadores como sucedería varios siglos después. El mundo impreso, incluyendo también los medios impresos, generó tarde centros de documentación para conservar las informaciones que se publicaban y que tendrían un valor a lo largo de la historia. Sí, al menos, hubo un interés por su conservación, no tanto por su análisis, de ahí que en la actualidad algunos periódicos centenarios, como $A B C$ y/o La Vanguardia, dispongan de una hemeroteca digital, porque antes tuvieron un archivo, en la que cualquier puede acceder a sus contenidos informativos.

\subsection{El mundo antes de Internet: poca y variada información}

En el siglo XX se rompe esa dinámica. Libros que encuentran millones de lectores; periódicos con incontables seguidores; emisoras de radio con audiencias millonarias; la televisión entrando en casas de todo el mundo. Producción para millones de audiencias, pero aún así el control seguía en manos de los principales grupos de comunicación.

Antes de la llegada de las Tecnologías de la Información (TI), algunos autores ya habían avisado de esta posibilidad. Tefko Saracevic predijo en los años sesenta que habría una "explosión de la información" y que sería difícil de asumir para 
poder ofrecerla a los autores, de tal manera que no perdiera vigencia. En un artículo posterior, de 1999, ofrece un estudio sobre el origen de la Documentación en relación con los problemas de la explosión de la Información, el papel social de la Documentación, la naturaleza de la información en la Documentación, la evolución del aprendizaje y enseñanza, la relación de la documentación con otras ciencias afines, modelos educativos, y la relación con la Biblioteconomía y la Informática. Es, precisamente, en esos dos frentes sobre todo en la informática donde se pone el acento para movilizar la información que ya se está produciendo en el mundo.

Autores de la talla de Burkhardt, MacDonald, y Rathemacher, vuelven a insistir más adelante en ese mismo concepto de Saracevic. Para ellos, se pasa de los primeros mensajes en la civilización, que se emitían para subsistir, que apenas viajaban unos pocos kilómetros, a la actualidad en la que la cantidad de información disponible se duplica cada siete u ochos años. De Solla Price, padre de la cienciometría, ya en 1956 destacaba el proceso de crecimiento exponencial que registra la información científica. En función del área de conocimiento, Price estimaba que la información científica se duplica cada diez o quince años, un ritmo de crecimiento que ya entonces resultaba muy superior al de la mayoría de fenómenos sociales. Desde sus estudios se constata un gran incremento en la velocidad del ritmo de producción de información. Muchos expertos consideran que se ha producido más información en los últimos 10 años que en todos los siglos anteriores y el caudal es tan largo y extenso que ya nunca se podrá leer ni controlar todo lo que se publica en el mundo, aunque la ciencia haya logrado alargar la vida más allá de los ochenta años de media.

Hace ya más de una década, en otro artículo de esta misma revista ${ }^{2}$, se planteaban estos problemas que bibliotecas y centros de documentación trataban de resolver: el creciente volumen de publicaciones. Y desde entonces, no hizo sino empeorar. El investigador Angulo Hoyos publicó un texto que indicaba claramente las tendencias de la información: "En los últimos 10 a 15 años se ha escrito y publicado más que en toda la historia de la humanidad. No menos de 55.000 libros nuevos son editados cada año, y se publican en el mismo período más de dos millones de artículos nuevos en por lo menos 65.000 revistas técnicas, aumentando un depósito anterior de unos 50 millones de artículos" (Hoyos, 1999) ${ }^{3}$. Una década después, las cifras han crecido tanto que lejos de simplificar han complicado más el panorama de la producción científica, la educativa, la de los medios de comunicación; en definitiva, el conocimiento en todas sus facetas.

${ }^{2}$ Marcos Recio, Juan Carlos. "Desarrollo de aplicaciones documentales: ¿para qué sirve la información en una sociedad global?", en Documentación de las Ciencias de la Información. Madrid: Editorial Complutense, Vol. 22, 1999, pp. 13-25.

3 Ángulo Hoyos, Enrique Carlos. Observatorio de nuevas tecnologías. http://www.serweb.com/andr-5.html 
Hay un antes y un después de los años sesenta. Hasta entonces, la mayoría de los científicos eran capaces de asumir, controlar, leer y descifrar la información que se producía en el mundo. Había un control y un seguimiento de la ciencia. Pero el crecimiento exponencial de la misma, desbordó todas las previsiones y supuso un desconocimiento para los científicos, ahora incapaces de gestionar los textos y publicaciones que aparecían en el mundo. Crecían las publicaciones, aumentaban los científicos, el mundo tenía más publicaciones y los medios de comunicación más lectores y oyentes. El mundo dejó de ser impreso para acercarse por primera vez al mundo digital, pero aún quedaba al menos una década y el nuevo descubrimiento de características similares a la imprenta: Internet.

\subsection{EI mundo con Internet: la información con datos}

Resulta prácticamente imposible cuantificar el número de informaciones que se publican cada día, el número de documentos que se generan, los estudios, los análisis, en definitiva el conocimiento. Esta explosión informativa ha dado como resultado un enorme incremento en el número de personas dedicadas a manejarla, analizarla e interpretarla. La función del bibliotecario y del documentalista ha dejado paso a la del gestor de la información. Se trata de una figura que rastrea las redes en busca de la mejor información, la selecciona, la analiza y la facilita en un tiempo récord, porque en la actualidad la toma de decisiones, sobre todo en el mundo empresarial requiere de esa rapidez en la difusión.

Fue a comienzos del siglo actual cuando se hicieron los primeros estudios para cuantificar la información. La cantidad de datos en el mundo crece exponencialmente, por eso se hizo cada vez más difícil saber el número total de contenidos que circulaban por las redes. No son cifras exactas, son estudios científicos, pero los resultados son siempre aproximados.

El estudio más importante, realizado durante varios años, parte de una investigación que se lleva a cabo en la universidad de Berkely por parte de Hal Varian y Peter Lyman entre 2000 y 2003: "How much information". Los resultados del estudio señalaban que cinco exabytes de nuevos datos fueron almacenados a nivel mundial en 2002, y que se transmitieron 18 exabytes. También estimaron que la cantidad de nuevos datos almacenados se duplicó de 1999 a 2002. El seguimiento se hizo en cuatro niveles: papel, periódicos, revistas y libros; cine y soportes audiovisuales; magnéticos, con discos duros, memorias ópticas, etc. y ópticas con cd y dvds.

Un segundo estudio lo realizó la firma de investigación IDC. Se trata de una serie de informes anuales sobre el universo digital. El primero se llevó a cabo en 2007, patrocinado por EMC. Ya entonces había más empresas trabajando en el estudio de datos, como señala Dan Veste ${ }^{4}$, vicepresidente de programas, soluciones de Business Analytics de IDC: "El mercado de los grandes datos se está

${ }^{4}$ The Daily Telegraph (2012). How much information? Some landmark research has helped us to quantify just how big is big., 17 November 2012 
expandiendo rápidamente a medida que las grandes empresas de TI y nuevas empresas compiten por los clientes y la cuota de mercado" The Daily Telegraph, 2012).

El último estudio que aportó una visión amplia fue el que Martin Hilbert y Priscila López publicaron en la revista Science. En dicho artículo se explicaba en detalle que la capacidad de almacenamiento mundial creció a un ritmo anual del 23 por ciento desde 1986 hasta 2007 y que el almacenamiento digital de la información estaba ocupando el terreno a la información analógica.

Pero la información también tiene un valor económico, ya que los datos sirven como sustento de la economía mundial y como nuevas fuentes de inversión, ayudan al aumento de la productividad y la competitividad de las empresas, incluidas las públicas. El reto es manejar con acierto el gran volumen de datos que se crean. Por eso, los gestores de la información tienen en sus manos el reto de usar y conservar la información de manera dinámica y guardarla para el futuro de la sociedad.

\subsection{Las cifras de los medios, una perspectiva de futuro}

Mientras que se hacía el cambio de lo impreso a lo digital se fueron produciendo algunas transformaciones en los medios de comunicación, en especial en sus audiencias. Lectores fieles al mundo impreso se fueron reduciendo. Crecieron lectores con ganas de obtener información en ambos lados. Lo mismo les sucedió a otros, especialmente a Internet, donde lugares que no tenían una estructura de medios empezaron a contar con lectores/seguidores de ese sitio web.

Resulta del todo imposible, con las audiencias millonarias que han conseguido sitios de internet como Facebook, Twitter o LinkedIn, saber el lugar que ocuparán los medios de comunicación en el futuro. En este artículo se toma como referente el estudio: How Much Information? 2013: Report on American Consumers ${ }^{5}$, en el que se muestran las tendencias de los medios en un futuro inmediato. Los resultados obtenidos provienen de un análisis de más de 30 fuentes de datos de diferentes medios de comunicación, que van desde los medios tradicionales (TV, Radio, telefonía de voz) a nuevas fuentes digitales (tablets, dispositivos de juegos móviles, smartphones, vídeo móvil).

El consumo de medios seguirá experimentando un crecimiento en 2015. Según dicho estudio, se estima que: "Los estadounidenses consumen tanto los medios tradicionales y digitales con más de 1,7 billones de horas, un promedio de aproximadamente 15 horas y media por persona y por día. La cantidad de medios de comunicación superará 8,75 zettabytes por año, o 74 gigabytes" (Bohn; Short, 2013). El mencionado estudio contó además con dos consultoras de renombre como son Nielsen y ComScore, a través de declaraciones proporcionadas por las

${ }^{5}$ Bohn, R.; J. E. Short (2013). How Much Information? 2013: Report on American Consumers. San Diego, American Consumers, 2013. 
compañías de medios y los analistas, lo que da más valor al estudio, ya que ambas compañías son un referente en el estudio de medios, especialmente de audiencias.

Si nos centramos en el consumo de horas en los medios, televisión y radio (Figura 1), en el periodo comprendido entre 2008 y 2012, ambas siguen ocupando los primeros puestos para los usuarios, a pesar de que las redes sociales restan presencia en dichos medios. Aún así, la televisión perdió un $6 \%$ y la radio tan solo un $1 \%$.

Figura 1. Consumo de horas en medios de comunicación.

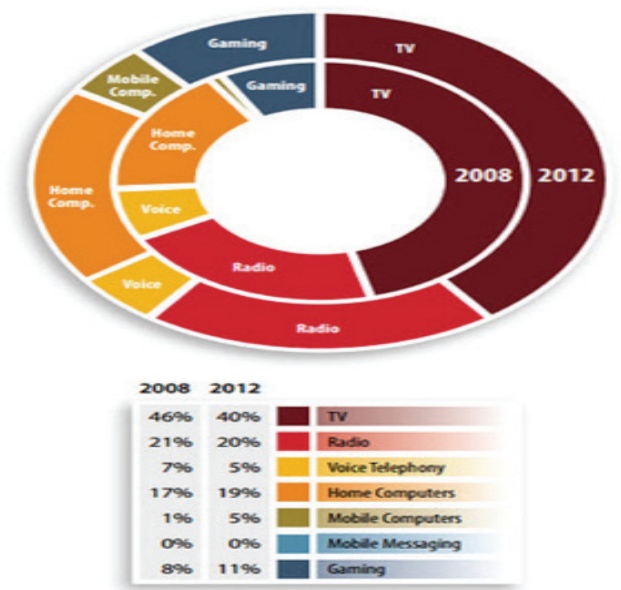

Fuente: How Much Information? 2013

Este gráfico resalta la importancia que siguen teniendo los medios de comunicación del siglo XX: radio y TV. Entre los dos suman el $60 \%$ de las horas consumidas. Sin embargo, también hay que destacar que más de la mitad de todos los bytes de los medios de comunicación son ahora consumidos por ordenadores, siendo el de los equipos móviles el segmento de más rápido crecimiento. En 2008, los dispositivos móviles representaron aproximadamente el 3\% de todos los bytes consumidos. En el año 2013 fueron un 10\%, lo que representa una tasa de crecimiento interanual del 27 por ciento.

Pero ¿qué sucede en España en estos medios, especialmente en TV? La actual situación económica que atraviesan las familias españolas está incidiendo en un mayor consumo de horas de televisión. Los consumidores disponen de más tiempo, ya que ha descendido el número de personas que van al cine y/o al teatro y otras actividades culturales y, en consecuencia, aumentan su tiempo de ocio frente a la televisión. Así, el consumo medio de televisión en España, durante 2011, "se situó en 239 minutos por persona y día, sobre una muestra de 44 millones de individuos mayores de cuatro años. Este dato supera en trece minutos el consumo de 2009 y en cinco el de 2010, pero es inferior en 28 minutos al récord histórico de 267 minutos registrado en febrero de 2012", según datos del Estudio de Egeda 2012. El 
crecimiento es imparable desde el año 2002, pero ha sido especialmente alto en los años de crisis como la actual (Gráfico 1).

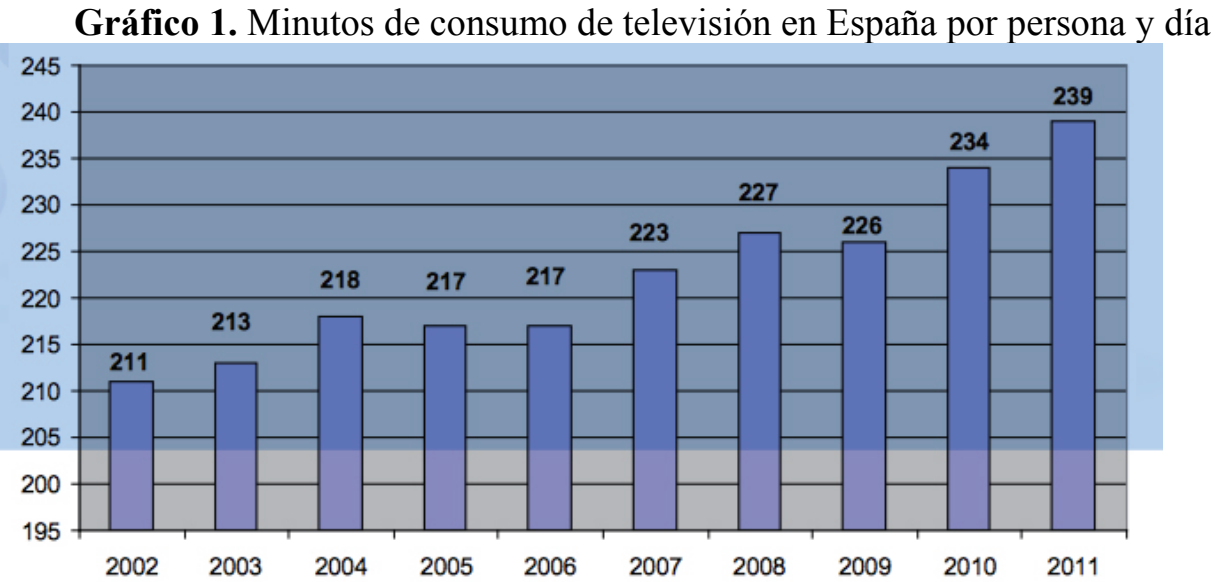

Fuente: EGEDA 2012.

Este es un reto difícil aún para los medios digitales, si bien ya hay distorsiones sobre ese consumo, sobre todo en publicidad, donde el grado de atención a lo que la televisión ofrece ha ido decreciendo. Se entiende como normal que los jóvenes mientras están frente a la televisión, que no es lo mismo que ver la televisión, tienen abierto su teléfono y están chateando o viendo una serie en ese dispositivo. Las audiencias se diversifican y los entornos finales hablan de dispersidad, frente a concentración. La televisión sigue sumando horas, pero no resultados para sectores como la publicidad.

Por su parte, el estudio How Much Information? 2013: Report on American Consumers recoge también el crecimiento en el total de horas anuales de los hogares de los Estados Unidos, empleando todas las fuentes desde 2008-2015 (Figura 2). Se midió el tiempo de consumo y el rendimiento de bytes de los datos facilitados por las personas encuestadas. De todos ellos, hay que resaltar como interesantes el avance del consumo en el vídeo. Así se recoge en el estudio: "Las fuentes de vídeo dominan el consumo de bytes, con 3,8 zettabytes procedentes de la televisión y 2,46 zettabytes de juegos de ordenador. Si se utilizan las horas como medición, los medios consumidos son de más amplia distribución, con cantidades sustanciales en la radio, aplicaciones de Internet, como redes sociales, navegación y búsqueda, y otros, incluyendo las comunicaciones de mensajería y correo electrónico". (Bohn; Short, 2013). 
Figura 2. Incremento anual en horas 2008-2015.

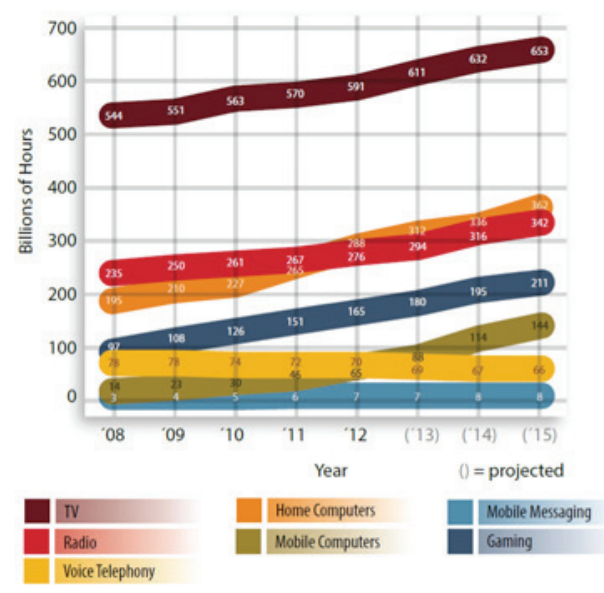

Fuente: How Much Information? 2013

Estos datos siguen ponderando la televisión como medio en el que se consumen más horas. La radio también está bien posicionada, pero el resto de medios ha ido perdiendo cuota que han sumado otros soportes, especialmente ordenadores y más en concreto el móvil. Un sector que avanza de manera considerable son los juegos, ya que hay una capa de la población que consume juegos al mismo tiempo que utiliza otros medios de comunicación. Entretenimiento e información y juegos es la suma de los tres vectores, independientemente del soporte, que más resultados están cosechando por parte de los consumidores.

Pero la mayor transformación de los medios está en los dispositivos móviles. Las cadenas de televisión, conscientes de esa necesidad, están adaptando sus formatos a este soporte clave de la comunicación. Los teléfonos del futuro, smarthpones, android, iphone libran una batalla para convertirse en el soporte principal para el consumo de tv, de juegos y de música. La figura 3 muestra un recorrido desde el año 2008 al 2015, en el que la comunicación sigue ocupando el lugar principal, pero en el que la música y los juegos tienen un importante papel de consumo semanal a través de dispositivos móviles. 
Figura 3. Consumo en horas semanales 2008-2015.

\begin{tabular}{|c|c|c|c|}
\hline & $\begin{array}{c}2008 \\
\text { Hours per User / Week }\end{array}$ & $\begin{array}{c}2012 \\
\text { Hours per User / Week }\end{array}$ & CAGR \\
\hline \multicolumn{4}{|c|}{ Feature Phones } \\
\hline Communications & 1:59 & $3: 37$ & $16.20 \%$ \\
\hline Web browsing and search & $1: 03$ & $2: 13$ & $20.50 \%$ \\
\hline Social networking & $0: 28$ & $2: 20$ & $49.50 \%$ \\
\hline Gaming & $0: 35$ & $1: 38$ & $29.30 \%$ \\
\hline Music & $1: 10$ & $1: 45$ & $10.30 \%$ \\
\hline Total Time & $5: 15$ & $11: 33$ & $21.80 \%$ \\
\hline Number of Users & $99,992,693$ & $81,737,510$ & $-4.90 \%$ \\
\hline \multicolumn{4}{|c|}{ Smartphones } \\
\hline Communications & $1: 59$ & $3: 37$ & $16.2 \%$ \\
\hline Web browsing and search & $1: 18$ & $3: 06$ & $24.3 \%$ \\
\hline Social networking & $0: 35$ & 3:09 & $52.4 \%$ \\
\hline Gaming & $0: 43$ & $2: 12$ & $32.4 \%$ \\
\hline Music & $1: 10$ & $1: 45$ & $10.3 \%$ \\
\hline Total Time & $5: 46$ & $13: 49$ & $24.4 \%$ \\
\hline Number of Users & $21,998,392$ & $88,548,970$ & $41.6 \%$ \\
\hline
\end{tabular}

Fuente: How Much Information? 2013

Esta lucha por las audiencias está en una fase experimental en la que los móviles son los mejor situados. Queda por saber cómo encajarán el golpe las cadenas de televisión. La radio en internet está funcionando a buen nivel y los periódicos no acaban de encontrar su hueco. En este sentido, se analizan a continuación cuatro propuestas que pueden ser referentes para los próximos años.

\section{LOS MEDIOS DIGITALES SE RESISTEN A DESAPARECER}

Mientras los periódicos digitales españoles gastan sus fuerzas en la búsqueda de plataformas que les reporten beneficios con la creación de redacciones integradas, que luego desintegran y en contenidos digitales que apenas consideran el valor que tiene el vídeo, especialmente la apuesta por el vídeo corto de 11-16 segundos. En Estados Unidos, diversos proyectos están siendo referentes para crear comunidades informativas que esperan de equipos reducidos contenidos de primer nivel. No hay que olvidar que al frente de esos proyectos figuran periodistas de raza, curtidos en experiencias impresas y digitales, especialmente estas últimas en medios poderosos que han aprendido a base de acierto-error.

La palaba clave de todos estos proyectos es movilidad. La información, concebida a la manera tradicional, ya murió. Los contenidos digitales requieren de apoyos gráficos y multimedia, fundamentalmente historias en vídeo, pero también algunas temáticas que los medios generalistas olvidaron o no tienen en cuenta. Los 
procesos de creación y difusión de la información dentro un mundo global están dejando paso a contenidos y materias especializadas, contadas por expertos periodistas que creen en lo que escriben y que no se sienten atados a una línea editorial y/o a una presión económica de la empresa para la que trabajan.

Cansados de publicar sin que nadie los lea; olvidados en un mundo digital al que muy pocos acceden, esperando la gloria de tiempos pasados y descubriendo que las formas ya no importan tanto, el nuevo periodista está más cerca del lector cuando detecta sus necesidades y trabaja de manera directa para él. Los problemas no están situados a miles de kilómetros. La información requiere de un compromiso social de quien la escribe, aunque parezca que nunca le va a afectar. No hay una información principal y varias secundarias, ni un tema más importante que otros. Los lectores de medios digitales valoran aquella información que suma para ellos, que confía en lo que ellos aportan y sobre todo que les coloca en el centro de la actividad informativa.

Durante mucho tiempo, los periodistas han atendido los cantos de sirena de multinacionales, grupos de poder, entornos mediáticos, gentes con poder económico y se olvidaron de sus lectores. Principio básico del periodismo lo fue y lo será que el periódico existe porque hay lectores. Entonces, ¿por qué nos hemos olvidado de ellos? Durante la primera década del siglo XXI, se les dio la oportunidad a través del periodismo ciudadano para que publicaran en los medios. Incluso muchos de ellos crearon algún blog con repercusiones importantes en las audiencias. Luego, a una minoría se le incluyo ese blog dentro de un medio general de información. Pero esto no ha sido suficiente, o no lo es para entender la caída de lectores que se está produciendo cada año en los medios, incluidos los digitales, enfrentados a una crisis multifactorial de enorme complejidad.

Hay quien considera que la causa principal es que los lectores se han convertido al sistema multimedia, que la lectura es algo caduco y que si lo pueden entender en 20 segundos, porqué estar leyendo 20 minutos. Lejos de inquietar al lector, los medios digitales han sido capaces de crear nuevos contenidos en base a lenguajes digitales, más dinámicos y con mejores datos documentales. En apenas una década, el lenguaje informativo, incluidas las cadenas de televisión, adoptan elementos multimedia y dejan para la reflexión la lectura más pausada, esa que ayudará a crear una opinión más sustentable. Si se hiciera un estudio sobre la manera en que los ciudadanos ven los medios, un porcentaje muy alto respondería con algunos detalles sobre lo que vio en el último informativo de la TV. Si por la mañana se preguntara a esos mismos ciudadanos, la radio les habría servido para responder. ¿Y el resto del día? Deberían ser los periódicos, pero ya no lo son. La cultura audiovisual está creando ya la cultura visual del consumidor de medios. No hay reflexión crítica, ni lectura crítica porque los medios escritos son considerados enemigos de la visión general que los lectores tienen de las noticias. Sólo en ocasiones muy especiales, se acercan a descubrir por sí mismos, a crearse una opinión en base a otras lecturas de las que el periódico impreso y/o digital aporta a sus lectores. El problema no es único de los medios de comunicación escritos, lo es 
de las bibliotecas, de los centros de comunicación, de la lectura, del teatro, del cine, en definitiva de la cultura, del predominio de la cultura audiovisual sobre la escrita.

Pero los medios digitales se resisten a desaparecer. Lo hacen por la insistencia de algunos periodistas, a título individual, o en pequeños grupos de redactores, que detectan un nicho de mercado no cubierto por los grandes medios y se apropian de él. El periodismo cambia por las necesidades del lector, que empuja a los medios a ofrecer lo que desean, no lo que antes le entregaban en formato papel. Los editores siguen creyendo que el periodismo es el referente informativo para una sociedad más justa y democrática y que en consecuencia deben existir periódicos que den fe de esa sociedad. Pero no es menos cierto que la opinión ya no solo la crean los medios, sino que cada individuo es capaz de sumar muchas informaciones y crear la suya. Ahora ya no depende de los medios como hace dos décadas. El consumidor de noticias es independiente para tomar decisiones, sobre todo si dispone de conexión a Internet y es un lector activo, crítico y reflexivo de los medios digitales. Ahora el reto es la información elaborada en nuevos medios, dirigidos a audiencias especializadas.

\section{CUATRO MODELOS DE MEDIOS DIGITALES, UNA PROYECCIÓN A TENER EN CUENTA}

Con frecuencia, la mayoría de estudios que se publican en revistas como ésta hacen un balance de medios que ya están funcionando o que han logrado alcanzar altas cuotas de audiencias. Se piensa que hacerlo sobre proyectos que aún no se han consolidado es arriesgado, pero no lo es tanto cuando sirven para que futuras publicaciones tomen en cuenta los ensayos que se están realizando. Los "cementerios informativos" están llenos de medios que nacieron con gran energía y vitalidad y que al poco tiempo desaparecieron. Se muestran cuatro proyectos en entornos digitales con periodistas de renombre al frente de los equipos de redacción. Estos proyectos tienen una idea común: dar un servicio a determinadas comunidades que no reciben todo el trato informativo que merecen, sean temas de justicia, de comunidades aisladas y/o de lectores que no buscan especialmente un beneficio directo. El periodismo ciudadano está dejando paso al periodismo de contenido social, apoyado en noticias inmediatas, urgentes y con amplia participación de la audiencia. Estos proyectos están capitaneados por periodistas que han trabajado en medios importantes, The New York Times, The Washington Post, etc., y que libremente dejaron su cómodo puesto de trabajo para enrolarse en un nuevo tipo de hacer periodismo, arriesgado y comprometido.

Aquí se muestran cuatro propuestas que han sido bien acogidas entre los lectores de todo el mundo, especialmente los norteamericanos: uno, First Look Media, un proyecto de 250 millones de dólares capitaneado por el fundador y presidente de Ebay, Pierre Omidyar; dos, Vox Media, una idea del siempre 
poderoso Esdras Kein; tres, Proyecto Marshall, de Bill Keller y cuatro Mashable, de Brian Ries.

\subsection{First Look Media, Pierre Omidyar https://firstlook.org}

Estos cuatro proyectos arrancan con el compromiso de un cambio informativo, paralelo al que están experimentando los medios tradicionales, pero con una implicación mayor por parte de las audiencias. No se crean grandes redacciones, ni se apuesta por opiniones excesivamente técnicas; lo que se pretende es informar de manera ágil y contar con los comentarios y participaciones de los lectores. Hay que destacar también, en cada uno de ellos, la/s persona/as que han iniciado los proyectos, inversores y expertos en medios digitales que ahora aportan su conocimiento al mundo de la información.

Figura 4. Logotipo de First Look Media.

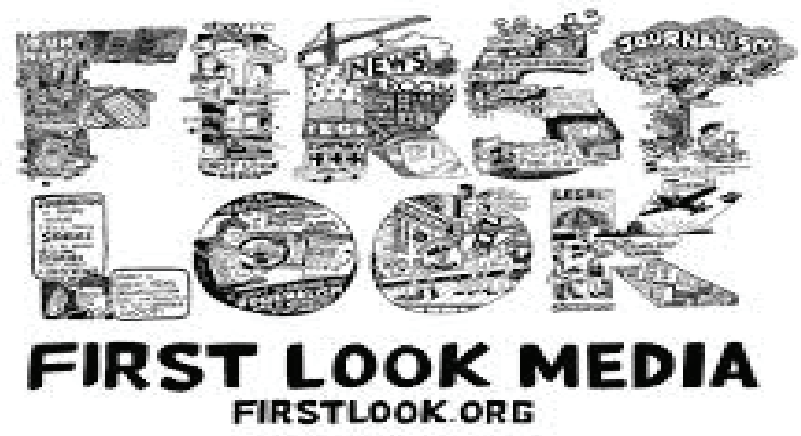

Fuente: https://firstlook.org

First Look Media, según se indica en su sitio web https://firstlook.org/ "pretende reinventar el periodismo en la era digital, la combinación de la promesa de la innovación tecnológica con el poder de la información sin miedos"6. Este medio, fundado por Pierre Omidyar, apuesta por un periodismo de rigor; en palabras de su fundador: "periodismo original, independiente, que está profundamente informado e investigado, bien hecho, comprobado y contrastado, y muy bien dicho". En principio, nada nuevo, porque esos son los elementos que han movido al mundo de la información desde hace al menos dos siglos. Sin embargo, hay una parte de valor añadido que hasta la llegada de las redes sociales no se producía, como es la participación ciudadana: "Nos impulsa sobre todo por la creencia de que la democracia depende de una ciudadanía que no está sólo altamente informada, sino profundamente comprometida". Aunque en sus principios contempla los elementos fundamentales del periodismo; en la actualidad, la respuesta por parte de los lectores a informaciones manipuladas o presentadas sin suficientes datos significaría una avalancha de respuestas y un desprestigio del propio medio. Por eso, su compromiso con los lectores para por:

\footnotetext{
${ }^{6}$ Cfr. https://firstlook.org
} 
"En todo nuestro trabajo, estamos comprometidos con los estrictos estándares de exactitud y la honestidad, la voluntad de informar de nuestros propios errores e inconsistencias, así como las de los demás, y un profundo respeto por el poder transformador de las historias verdaderas".

First Look Media pretende ser una gran plataforma de periodismo digital que englobará diferentes medios especializados en información política, económica, deportiva, social, tecnológica, etc. Aunque se ha constituido como una organización sin ánimo de lucro se plantean como objetivo prioritario lograr la viabilidad económica a través de diferentes ingresos para de esa forma garantizar su total independencia. El proyecto está impulsado por Pierre Omidyar, empresario estadounidense fundador y presidente de Ebay. Omidyar ha realizado una inversión inicial de 50 millones de dólares para lanzar la plataforma y anuncia nuevas aportaciones en los próximos meses. Tras descartar la posibilidad de adquirir The Washington Post, decidió fundar una plataforma para realizar un periodismo totalmente nuevo. Omidyar llevaba años impulsando diferentes movimientos e iniciativas en favor de la transparencia informativa y el buen gobierno por parte de las administraciones públicas y empresas, que conectan con la filosofía editorial de su nuevo proyecto.

En First Look Media, Lynn Dombek, responsable del periodismo de investigación de Associated Press durante diez años, es el director de investigación de la organización. Además, el equipo directivo está integrado por periodistas de alto nivel, entre ellos los galardonados Glenn Greenwald, Laura Poitras y Jeremy Scahill.

En febrero de 2014 First Look Media presentó su primer medio digital: The Intercept https://firstlook.org/theintercept/. El editor jefe de la publicación es John Cook, anterior responsable editorial de Gawker. Este medio digital pretende materializar los principios configuradores que persigue Omidyar con First Look Media: convertirse en una bandera del periodismo de investigación independiente. En principio The Intercept surge como un medio para la difusión y el análisis de todos los documentos e informes filtrados de la NSA (Agencia de Seguridad Nacional de los EEUU). Se trata de una apuesta directa por el "periodismo de confrontación" con todos los ámbitos de poder que busca contribuir a una mayor transparencia y a denunciar todas aquellas malas prácticas que puedan ser constatadas. La redacción fundacional cuenta con dieciséis periodistas con amplia experiencia, procedentes de medios tradicionales y digitales.

Para finales de 2014, First Look Media anuncia el lanzamiento de una nueva publicación -todavía sin nombre- centrada en la denuncia periodística de la crisis financiera y las responsabilidades políticas escondidas tras ella. El director será Matt Taibbi, periodista especializado en información económica que ha trabajado entre otros medios en la revista Rolling Stone. De nuevo se buscará combinar el periodismo de investigación con la innovación tecnológica, los dos componentes que Omidyar considera transformarán por completo el periodismo y la comunicación en la nueva era. 
A pesar de las numerosas incógnitas que siempre implica un proyecto periodístico tan incipiente son muchas las fortalezas que hacen de First Look Media una iniciativa muy prometedora. En primer lugar, la presencia y la apuesta decidida de Pierre Omidyar, uno de los hombres más ricos del planeta y principal impulsor del comercio electrónico mundial. Omydiar, considerado como un visionario de Internet, se muestra totalmente entusiasta ante las nuevas posibilidades del nuevo periodismo en el mundo digital. En segundo lugar, su sólida estructura organizativa que combina la presencia de periodistas de primer nivel y grandes expertos en los negocios digitales. Y, por supuesto una concepción muy clara del estilo periodístico y los objetivos que deben perseguir sus publicaciones.

\subsection{Vox Media, http://www.voxmedia.com/ jim bankoff}

Figura 5 Logotipo Vox Media

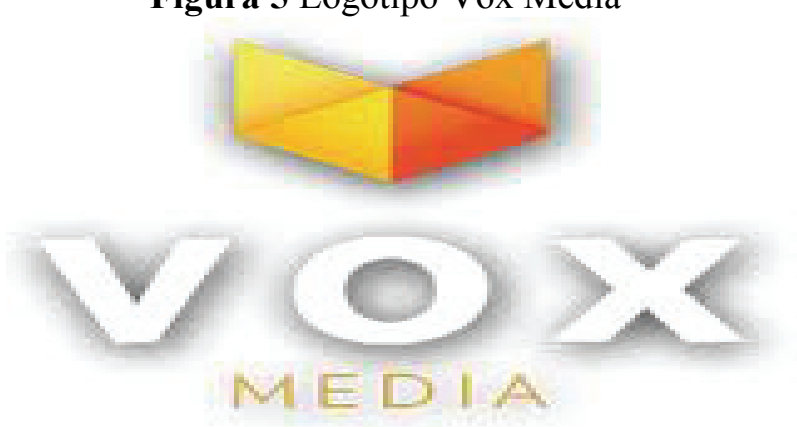

Fuente: http://www.voxmedia.com/

Este segundo proyecto es más integral que el primero. En realidad se trata de un sitio de noticias moderno que busca un contexto concreto, con importantes explicaciones a nivel informativo, con una cartera de editores digitales que incluye actualmente seis medios diferentes: The Verge, SB Nación, Polygon, Curbed, Eater, Racked y Vox. Fundado por Jerome Armstrong, Tyler Bleszinski y Mankos Moulitsas, Jim Bankoff es su consejero delegado.

¿Cuál es su razón de ser? ¿Qué ofrecerán estos medios que no se publica en otros ya creados y con amplias audiencias? En Vox Media apuestan sobre todo por marcas de medios que atraen a la generación por cable o Wi-Fi. Este medio es un arte en sí mismo, tal como lo fue la TV, la radio y la prensa escrita. Para lograrlo cuentan con un equipo de "periodistas talentosos y narradores que saben cómo hacer participar a un público que, ante todo, consumen sus medios de comunicación en la web y el móvil’”. Apuestan por importantes herramientas con el fin de "decir la verdad" y trabajar con: "aplicaciones nativas de las muchas

\footnotetext{
${ }^{7}$ Cfr. http://www.voxmedia.com/
} 
herramientas de gran alcance (datos, sociales, multimedia, por nombrar algunos) que ofrece el medio digital.

"Estamos lanzando Vox.com para resolver un problema en el negocio de las noticias. Hemos pasado mucho tiempo en los medios de comunicación enterrando lo importante de la información. Hemos tomado ventaja con la velocidad de la web, pero no su profundidad o persistencia" ${ }^{8}$ Pero este tipo de periodismo, de nuevo periodismo digital, frente a los ensayos realizados en los cinco últimos años en otros medios, supone un compromiso de todos los miembros de la redacción y también de los lectores, a lo que se les pide ayuda para cambiar esta forma de comunicar: "Queremos construir una guía de usuario para las noticias producidas por los expertos dentro de un área que ellos conocen mejor. Vamos a tener una cobertura regular de todo tipo de informaciones, desde la seguridad fronteriza hasta lo que sucede en la ciudad. En lugar de dejar que los informes se llenen de polvo en un archivo, vamos a crear las contribuciones atemporales e interminablemente actualizadas. En lugar de decirle a los lectores simplemente lo que acaba de pasar, queremos decirles lo que es importante acerca de esa información y porque suceden esas cosas. Haremos -nuevas informaciones- entretejiendo palabras, vídeos, gráficos, visualizaciones de datos, aplicaciones de noticias y fotografías"”.

En realidad este proyecto es una amalgama de varios medios que unen sus fuerzas en determinados sectores de la comunicación; entre ellos: SB Nation (deportes), The Verge (tecnología/cultura), Polygon (videojuegos), Eater (gastronomía), Racked (moda y compras) y Curbed (decoración y vivienda). Y lo hacen porque creen que las audiencias son similares, con experiencia en la red y en los contenidos que les suministran.

SB Nation integra un total de 308 blogs y aglutina la mayor red de comunidades deportivas. Su editor jefe nacional es el periodista deportivo Spencer Hall. Cuenta con 22,1 millones de usuarios únicos, con un $40 \%$ del tráfico total generado desde dispositivos móviles. $S B N$ representa uno de los mejores exponentes de lo que implica la integración multimedia en la Web.

The Verge es un portal fundado en el año 2011, cuya temática es la tecnología, la ciencia, el arte y la cultura. Desde sus inicios ha destacado por unos contenidos especializados que se caracterizan por la información de calidad y los reportajes en profundidad.

Polygon, fundada en 2012, es una web sobre videojuegos que intenta convertirse en el lugar de encuentro para los aficionados a los videojuegos y también para sus creadores.

Eater es un portal gastronómico con información sobre restaurantes y productos de alimentación. En 2005 inició su actividad en Nueva York y actualmente cuenta

\footnotetext{
${ }^{8}$ Vox Media. Data / visual / news app reporters. http://sbnation.theresumator.com/apply/4HIL1W/Data-Visual-News-App-Reporters.html

${ }^{9}$ Vox Media. Data / visual / news app reporters. http://sbnation.theresumator.com/apply/4HIL1W/Data-Visual-News-App-Reporters.html
} 
ya con veintidós ediciones locales en las principales ciudades de EEUU y con una edición nacional. Además de información y crítica gastronómica, cuenta con un potente buscador de restaurantes y lugares de ocio. Dentro de la política de contenidos se otorga una gran importancia a los espacios para la participación de los usuarios.

Racked es un medio digital especializado en información sobre moda y últimas tendencias. Sigue el modelo de explotación de los contenidos a través de diferentes ediciones para garantizar la cercanía con el usuario final. Además de la edición nacional se actualizan seis ediciones más para diferentes ciudades de los EEUU.

Por último Curbed está especializado en decoración y vivienda. Como producto del grupo VoxMedia sus claves de éxito son la especialización temática, el cuidado del diseño y la información de proximidad. En marzo de 2014 cuenta con 13 ediciones diferentes con información de las respectivas ciudades y zonas.

Para finales de 2014 VoxMedia ha anunciado el lanzamiento de un nuevo portal Vox, que en principio se dio a conocer como Project X, en espera de hacer público el nombre definitivo. El objetivo es ofrecer información contextual que a los usuarios les facilite comprender la información que todos los días reciben.

El planteamiento estratégico de este grupo -que en breve integrará a siete medios diferentes- es generar contenidos de gran calidad y utilidad para sus usuarios y anunciantes. Los propios promotores han autodefinido el proyecto como un intento de llevar al entorno digital lo que ha supuesto Condé Nast en el mundo editorial. Todos los medios que integra Vox Media comparten su propia plataforma de publicación digital llamada Chorus, sin duda una de sus principales señas de identidad que les permite un crecimiento armónico.

Como los proyectos anteriores, en fase experimental, no tienen claro aún su modelo de negocio final, pero sí el valor que hay que dar a las audiencias y a los anunciantes, tal y como se insiste en su sitio web: "A diferencia de los portales y periódicos, creemos que las marcas verticales (cuando se hace bien) tienen una autoridad de mando con las audiencias y los anunciantes que los sitios generalistas no hacen. Aunque desde un punto de vista empresarial, tenemos enormes eficiencias de nuestro producto compartido y plataformas de negocio".

\subsection{Proyecto Marshall, Bill Keller: http://www.themarshallproject.org/}

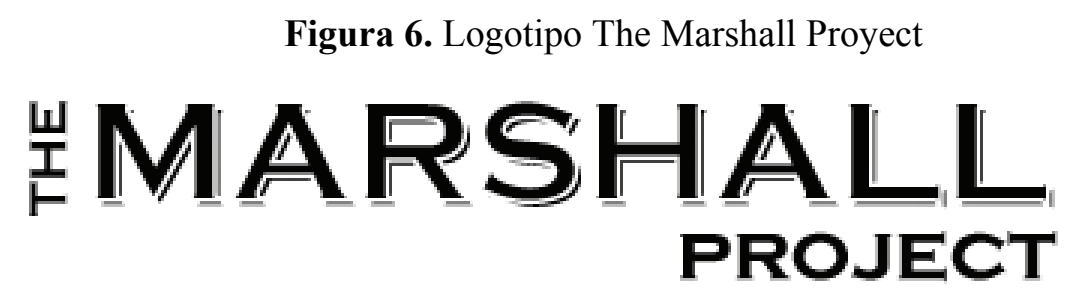

Fuente: http://www.themarshallproject.org/

El Proyecto Marshall arranca inicialmente con 20/25 periodistas a tiempo completo, con el apoyo de donaciones, de fundaciones y particulares. Se convierte 
en un medio de comunicación en línea que se enfrentará a las deficiencias del sistema de justicia penal en los Estados Unidos. Tiene prevista su salida a mediados de 2014. El nombre del proyecto es un homenaje al juez de la Corte Suprema de Justicia de los EEUU, Thurgood Marshall.

¿Qué pretende esta publicación? ¿Cómo se enfrenta al seguimiento informativo diario? No es un periódico a la manera tradicional. Busca otras maneras de ampliar la información, manejando fuentes documentales y colaborando con instituciones implicadas en el área que defienden. Así lo especifican al menos en su sitio web: "El Proyecto Marshall tendrá un pequeño grupo de periodistas, y también puede trabajar en colaboración con otras organizaciones de noticias. Además, tenemos la intención de sumar lo mejor de otros informes y hacer comentarios sobre el tema, incluyendo las voces de quienes están en el sistema. Tenemos la intención de hacer un gran uso de las herramientas disponibles actualmente para la recopilación de información, contar historias y llegar a un público más amplio, incluyendo vídeo, audio, gráficos, etc."10 Es un medio que sigue en construcción, creando una plantilla ágil, dinámica y especializada. Mientras se redactan estas líneas se sigue formando el equipo y el último fichaje para la redacción ha sido, según informa Bill Keller, editor en jefe del proyecto: Gabriel Dance, el galardonado editor interactivo de The Guardian EE.UU, quien se unirá al Proyecto Marshall como jefe de redacción. "Gabe Dance es una de las periodistas más creativas que conozco, un maestro de la narración de la era digital" "11, comentó Keller. En efecto es uno de los periodistas más galardonados en el ámbito digital con premios como un Emmy y un Alfred L. Dupont en 2011, varios premios de la Asociación de Noticias Online, seis premios Malofiej y seis de la Society for News Design.. También cuenta con un World Press Photo.

A través de un periodismo de profundidad y de investigación especializado en asuntos judiciales, The Marshall Project busca generar debate sobre los problemas de la justicia y el sistema penitenciario de los EEUU. En su página asegura que persiguen generar un movimiento en favor de la reforma de la justicia penal que trascienda en la campaña presidencial de 2016.

Además de ofrecer nuevos enfoques e informaciones propias pretenden convertirse en un lugar de referencia en la red para que los usuarios puedan consultar las noticias más relevantes sobre los temas judiciales del país.

Estos medios, también este proyecto, inciden sobre todo en ofrecer una información imparcial, algo que cada vez les cuesta más a los grandes medios digitales, influenciados por su línea editorial e intereses políticos, religiosos, financieros y publicitarios. Buscan con su información una total claridad y trasparencia informativa para su audiencia y contrario a los grandes medios, por

${ }^{10} \mathrm{Cfr}$. http://www.themarshallproject.org/

11 The Marshall Project. Gabriel Dance joins The Marshall Project as managing editor. http:/www.themarshallproject.org/announcements/gabriel-dance-joins-the-marshallproject-as-managing-editor/ 
ejemplo The Times o de otras organizaciones de noticias, el Proyecto Marshall ofrecerá un enfoque más allá de lo que hacen los grandes medios que tienen determinadas limitaciones.

Quizás la mejor opción informativa que ofrecen este tipo de proyectos es lo que se llama: "noticias sin ánimo de lucro". Aunque este concepto es relativamente joven y no se ha aplicado en su totalidad, ya existen organizaciones que están teniendo éxito en la manera en que tratan las informaciones, sobre todo en temas de justicia, cárceles, sentencias judiciales y todo tipo de noticias relacionadas con los ambientes penales, que es donde este medio va a incidir más.

\subsection{Mashable, Brian Ries http://mashable.com}

El cuarto medio con repercusión digital es Mashable, una de las primeras compañías de medios con una fuerte presencia en redes como Twitter: $\mathrm{http}: / / \mathrm{www} . t w i t t e r . c o m$ dando a sus reporteros y editores la posibilidad de tener conversaciones en directo con la comunidad Mashable en torno a los acontecimientos y problemas que más les importan a los ciudadanos.

En su sitio web se definen como: "Una fuente líder de noticias, información y recursos para la generación conectada. Mashable informa sobre la importancia de la innovación digital y la forma en que potencia e inspira a la gente de todo el mundo" 12 . Este medio dispone de importantes audiencias y seguidores puntuales que acuden a su portal a leer algunos contenidos informativos. En concreto, según datos del propio medio, cuentan con 34 millones de visitantes únicos en todo el mundo y 14 millones de seguidores en las redes sociales, siendo una de las comunidades online más influyentes y comprometidos.

Esta compañía ya lleva unos años en el mercado, no es tan reciente como las anteriores, pues fue fundada en 2005. Tienes su sede en Nueva York y acaba de abrir una oficina en San Francisco. Se define a sí misma como: "La compañía de medios líder para la generación conectada y la voz de la cultura digital". El responsable de este proyecto editorial es Brian Ries, un experto en medios que trabajó para The Daily Beast, donde fue editor jefe y para la revista Newsweek. Junto a Ries trabajará como director de contenidos y Editor Ejecutivo, Jim Roberts que considera a este sitio web como un espacio en el que: "somos muy hábiles en la creación de historias que se pueden compartir y optimizarlas para la web social" (Prweb, 2014).

${ }^{12} \mathrm{Cfr}$. http://mashable.com 
Figura 7 Logotipo The Mashable

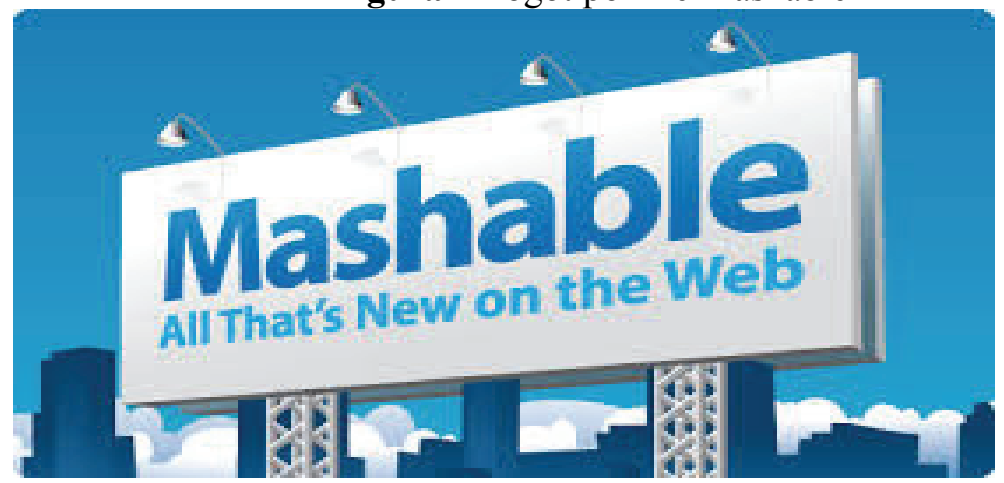

Fuente: http://mashable.com

Estos cuatro proyectos presentados, algunos más avanzados y otros que se implementarán a los largo de 2014, representan determinados retos y avances en los contenidos digitales que los medios de comunicación tendrán como competencia directa. Se trata, en realidad, de cubrir espacios informativos en la cultura, el deporte, la justicia, etc., que los medios tradicionales no llegan a profundizar; o no lo hacen, en la medida en que los lectores, ahora más activos, quieren.

La lista de proyectos es amplia. No se trata de hacer una relación extensa, pero sí de avanzar nuevas ideas, nuevos caminos para los medios digitales, dirigidos por periodistas de renombre y apoyados en expertos en contenidos digitales. Se enfrentan a nuevos retos, cuando ya tenían garantizada su permanencia en medios de gran prestigio profesional, pero buscan nuevas formas de hacer llegar la información a los lectores, ya con equipos de redactores más reducidos, pero con planteamientos abiertos, unificados y sobre todo ganando la confianza de sus lectores.

Queda por resolver el modelo de financiación que emplearán los editores de estos medios. Algunos de los presentados ya gozan de audiencias millonarias que atraen a publicitarios, pero se requiere en Internet nuevas maneras de hacer más eficaz la publicidad, como señala Ormaetxea. Que los medios digitales ocupen un poder privilegiado va a depender también de otros recursos, fuera de la publicidad: "El más notable tal vez es que ninguno confía en la publicidad digital tradicional para financiarse. Se están diversificando extraordinariamente las fuentes de ingresos y el comercio electrónico empieza a tener un significativo protagonismo" (Ormaetxea, 2014). Estas propuestas van a contribuir a reforzar los ingresos de estos medios. 


\section{CONCLUSIONES}

El cambio constante que están experimentando los medios digitales, abriendo un hueco significativo frente a los medios impresos, se traduce ya en formatos creados por grupos más reducidos de periodistas, acompañados de expertos en contenidos digitales que harán de esos lugares un encuentro de reflexión y participación activa de las audiencias. En este sentido, el presente trabajo parte de la situación actual en el consumo de medios digitales, incluidas radio y televisión para situar luego el entorno en el que se han de establecer estos proyectos.

Durante la primera década del siglo XXI los periódicos han ido experimentando con redacciones separadas para la versión impresa y digital, redacciones integradas, redacciones adaptadas a ambos formatos, etc., pero al final se ha demostrado que los contenidos digitales requieren no tanto del tamaño de la redacción, sino de la integración de sus miembros, por eso en los proyectos que se presentan, no se crean grandes redacciones, sino especialistas en la materia que sean capaces de generar contenidos digitales activos e interactivo.

Los medios digitales se han adaptado de diferentes maneras a los lectores. Hay una tarea común en los medios digitales: la participación de los lectores en los procesos de creación de contenidos. No en la elaboración de la información, que esa sigue en manos de los periodistas. Se trata, sobre todo, de integrar a los lectores, haciéndoles que crean en la información, que la refrenden con sus comentarios y que sean miembros activos de la comunidad informativa. Se espera de la audiencia una participación activa, lo que llevará a esos sitios a lugares preferentes en los medios digitales y, en consecuencia, a contar con otros recursos publicitarios frente a los medios masivos en audiencias millonarias. Se trata de calidad de la información, frente a la cantidad que ya ofrecen los medios digitales.

El proceso informativo también se somete a cambios importantes. Se busca, además de la calidad, la buena información, bien refrendada y avalada, con aportaciones de expertos en el área, incluso con los implicados directamente en esa comunidad. Todo con el fin de hacer sentir la información como algo más próximo. En los entornos globales, la información se vuelve más local para traspasar otras comunidades, otras fronteras.

Otra característica importante es el efecto social de la información. Crear noticias que interesan a la comunidad. Son las llamadas noticias sin ánimo de lucro. Informaciones que afectan de manera directa a una parte de la comunidad, pero que esa experiencia se puede luego trasvasar a otros países que han pasado o pasan por situaciones similares. Este reto social se está experimentando también en el llamado crowdfunding inverso, que consiste en ofrecer una información que interesa a la comunidad y que esa comunidad pague por ella, por lo que ha ahorrado al disponer de esa información.

$\mathrm{Y}$ en este recorrido hay que buscar un valor comercial a la información. Su producción y distribución requiere de un modelo de inversión menor, pero eso no significa que las pérdidas se mantengan largo tiempo. En algunos casos hasta tres 
años. El planteamiento es hacer una buena información para contar con lectores que a su vez estén interesados en participar y formar parte de esa comunidad. ¿Habrá publicidad? ¿Será efectiva? ¿Suficiente para hacer frente a los gastos? Estas preguntas aún no tienen respuesta, pero los diferentes proyectos presentados, así como otros que ya funcionan en las redes, han sido capaces de obtener beneficios para el pago de los periodistas y un pequeño margen de beneficio para los editores. Es tan solo el comienzo, pero ya es significativo que la inversión es menor, con resultados menores, pero más adaptados al medio y a los tiempos.

\section{REFERENCIAS BIBLIOGRÁFICAS}

ÁNGUlO HOYOS, Enrique Carlos. "Observatorio de nuevas tecnologías". (http://www.serweb.com/andr-5.html) Consulta: 03-03-2014.

BILTON, Ricardo. "Reality check: robots will not be writing the news", 27-032014. (http://digiday.com/publishers/robot-reporter-reality-check/) Consulta: 28-03-2014

BOHN, R.; SHORT, J. E. "How Much Information? 2013: Report on American Consumers". San Diego, American Consumers, 2013.

BURKHARDT, Johanna M.; Mac DONALD, Mary C.; RATHEMACHER, Andrée J. "Information Explosion", en Teaching Information Literacy, 35 practical standards-based exercises for college students, American Library Association, 2003.

EGEDA. Panomarama Audiovisual 2012. EGEDA. Entidad de Gestión de Derechos de los Productores Audiovisuales.

(http://www.egeda.es/documentos/PANORAMA_AUDIOVISUAL_2012.pdf) Consulta: 02-01-2014.

LYMAN, Peter; VARIAN, Hal R. "How Much Information 2003?” Universidad Berkeley.

MARCOS RECIO, Juan Carlos. "Desarrollo de aplicaciones documentales: ¿para qué sirve la información en una sociedad global?", en Documentación de las Ciencias de la Información. Madrid: Editorial Complutense, Vol. 22, 1999, pp. $13-25$.

MARCOS RECIO, Juan Carlos, SÁNCHEZ VIGIL, Juan Miguel, SERRADA GUTIÉRREZ, María. "Nuevos paradigmas periodísticos y documentales en los periódicos digitales: estudio de casos en España". México: Investigación Bibliotecológica, Archivonomía, Bibliotecología e Información, Septiembrediciembre, v. 23, núm. 49, 2009, pp. 43-65. ISSN: 0187-358X.

http://www.revistas.unam.mx/index.php/ibi/article/view/21390

"Mashable Announces Brian Ries as its Real-Time News Editor". New York, NY (PRWEB) February 11, 2014 (http://www.prweb.com/releases/2014/02/prweb11574687.htm) Consulta 1802-2014. 
OLIVERA ZALDUA, María; MARCOS RECIO, Juan Carlos; SÁNCHEZ VIGIL, Juan Miguel. "Estructura informativo-documental en los periódicos digitales. Análisis y cambios estructurales". Revista Mexicana de Ciencias de la Información. San Luis Potosí (México): Escuela de Ciencias de la Información (UASLP), vol. 3, núm. 1, sep-dic 2011 pp. 37-46.

Ormaetxea, Miguel. "El extraño caso de los medios digitales crecientes y la publicidad digital menguante", en media-tics.com 4/02/2014 (http://www.media-tics.com/noticia/4329/Medios-de-Comunicacion/El-extranocaso-de-los-medios-digitales-crecientes-y-la-publicidad-digitalmenguante.html) Consulta 06-02-2014].

SARACEVIC, Tefko. "Information Science", en Journal of the American Society for Information Science Vol: 50 Núm: 12 Páginas: 1050-1063, 1999

STELTER, Brian. Bill Keller's project: Focus on the 'scandalous' justice system. 10 febrero 2014 (http://money.cnn.com/2014/02/10/news/bill-keller-the-marshallproject/index.html) Consulta 18-02-2014.

"The Marshall Project. Gabriel Dance joins The Marshall Project as managing editor". http://www.themarshallproject.org/announcements/gabriel-dance-joinsthe-marshall-project-as-managing-editor/ Consulta 28-03-2014.

"The Daily Telegraph. How much information? Some landmark research has helped us to quantify just how big is big. 17 November 2012 (http://businesstechnology.co.uk/2012/11/how-much-information/) Consulta 2-03-2014.

Vox Media. Data / visual / news app reporters. http://sbnation.theresumator.com/apply/4HIL1W/Data-Visual-News-AppReporters.html Consulta 28-03-2014.

\section{Webgrafía}

The New York Times: www.nytimes.com/

The Washington post: www.washingtonpost.com/

First Look Media: https://firstlook.org/

Vox Media: http://www.voxmedia.com/

Mashable: http://mashable.com

The Marshall Project: http://www.themarshallproject.org/

The Intercept: https://firstlook.org/theintercept/

The Verge: http://www.theverge.com

Polygon: http://www.polygon.com

Eater: http://eater.com

Curbed: http://curbednetwork.com/specs

SB Nación: http://www.sbnation.com 